\title{
Optical Properties of Human Blood Sediment
}

\author{
B. Grzegorzewski* and E. Kowalińska \\ Biophysics Department, The Ludwik Rydygier Medical University in Bydgoszcz \\ 85-067 Bydgoszcz, Poland
}

\begin{abstract}
The formation of human blood sediment by means of optical method was studied. Light was transmitted through a layer of blood. The temporal changes of the transmitted light intensity along the sample were measured. Samples of blood from a healthy donor were investigated. The optical method permits us to distinguish three phases of the blood sediment. At the top of the container the well-known supernatant plasma layer creates. In the sediment of the red blood two phases were distinguished. The rouleau formation phase and the phase of demixed blood were found. The two phases are separated by the moving boundary. The kinetics of this boundary was determined. The optical data admit to a hypothesis about the physical phenomena of the sedimentation process. The spinodal decomposition may be one of the mechanisms of the blood sediment formation.
\end{abstract}

PACS numbers: 42.25.Bs, 82.70.Dd, 87.19.Tt

\section{Introduction}

Blood consists of plasma, gases, and cells. The composition of the complex fluid has a fundamental physiological significance [1]. A vide variety of elements of different nature and dimensions makes difficult to model the system. As a consequence of these difficulties approximate approaches in the study of blood are necessary. In the bio-colloidal model we assume that blood consists of plasma and erythrocytes. According to the classical definition the erythrocyte is a non-Brownian particle. The diameter of the erythrocyte is $8 \mu \mathrm{m}$ so that it is too large to exhibit thermal motion. However, mixing or an oscillatory shear flow causes an erratic movement of the particles. Intercellular bridges constitute attractive interactions between erythrocytes. The interaction causes that erythrocytes coalesce to form rouleaux [2-4]. With time the rouleaux build a chaotic three-dimensional network.

*corresponding author; e-mail: grzego@aci.amb.bydgoszcz.pl 
The intensity of the light transmitted by a blood sample increases the result of the rouleau formation $[5,6]$. The obtained curves are fitted by a sum of exponential functions and received parameters are used in the analysis of this rouleau formation kinetics. The attractive interaction between erythrocytes provides a necessary condition for a phase separation of the bio-colloidal system [7].

The sedimentation can be observed when a colloidal system is under the action of an external field. The translational velocity of Brownian particles is a manifestation of this phenomenon. The movement of the particles can be affected by interactions. Sedimentation process of an idealized colloidal system admits physical modeling. Many theoretical techniques can be used to analyze the effects that interactions have on the sediment formation [8].

The blood sedimentation phenomenon is utilized in clinical diagnostics since 1894 [9]. The monitoring of blood/plasma boundary position in time is the base of conventional investigations of the phenomenon. These conventional investigations deal with a global effect of the sedimentation process. In this way the erythrocyte sedimentation rate curve can be obtained [10]. Usually two straight lines fit this curve. It was shown that the second straight line of this curve corresponds to the sedimentation of already formed aggregates [11]. Recently the optical coherence tomography has been used in the study of blood sediment formation [12]. Changing the composition of the plasma one can obtain nonaggregating erythrocytes. In this case a deposit of erythrocytes occurs in the lower region of the blood sediment. In the upper region erythrocytes are in the erratic motion. Concentration profiles of the sediment and the kinetics of the moving boundary between the deposit and the phase of diffuse erythrocytes were experimentally and theoretically investigated $[13,14]$. The theoretical models of this sediment formation are insufficient to highlight the process even for the simplified system $[10,14]$.

To recognize physical phenomena associated with the blood sedimentation we have carried out the optical investigations. Blood of healthy volunteer was investigated. Measurements of the transmitted light intensity were performed. The recognition of mechanisms of blood sedimentation and properties of the blood sediment is achieved from the qualitative analysis of the optical data. The hypothesis about the phase separation associated with the sedimentation is taken into consideration.

\section{Material and method}

Blood from healthy voluntary was obtained by a venous puncture. To avoid coagulation the blood was treated with sodium citrate. The hematocrit of the original blood was $40 \%$. The samples with hematocrit $33 \%$ and $57 \%$ were obtained by the change of the amount of the plasma. Samples of uniformly distributed erythrocytes were obtained by gentle mixing before experiment. This blood was confined between two glass plates distanced by the teflon spacer. The thickness of 
the container was fixed to be $0.2 \mathrm{~mm}$ and the height was $20 \mathrm{~mm}$. The measurements were performed at room temperature. The experiment was carried out according to the ethical guidelines laid down by the Bioethical Commission at The Ludwik Rydygier Medical University in Bydgoszcz.

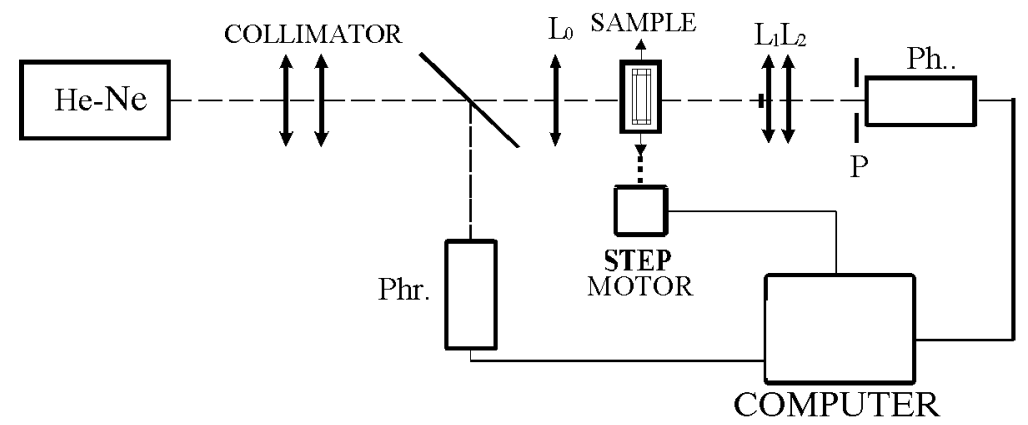

Fig. 1. Schematic diagram of the experimental set-up used in the study of the blood sediment formation.

The experimental set-up used for the measurements is shown in Fig. 1. The measurements were made using $\mathrm{He}-\mathrm{Ne}$ laser as the source of light. The laser beam after passing through the collimator was focused by the lens $L_{0}$. The container with the blood was placed at the beam waist position. The step motor moved the blood sample vertically with the constant velocity of $2 \mathrm{~cm} / \mathrm{min}$. The lens $L_{1}$ collimated the transmitted light and the lens $L_{2}$ focused the light in the plane $P$. The intensity of the transmitted light was obtained with a photomultiplier $P h$. The circular stop filter was situated in front of the lens $L_{1}$ to reduce the high intensity specular component of the light transmitted by plasma. The reference intensity was obtained with a photomultiplier $P h r$. The time varying signals were stored in the computer.

\section{Results}

Plots of the intensity of the light transmitted by the blood samples are given in Fig. 2. Coordinates of the horizontal plane of a particular plot determine the instantaneous location of the incident light beam along the sample. The dimensionless variable $x / l$ is the position coordinate in units of the height $l$ of the container. In these units 0 indicates the top of the container and 1 represents the bottom. The vertical axis represents the transmitted light intensity. Plasma and two phases of the red blood can be distinguished by a visual inspection of the plots. The high values of the intensity registered at the top part of the container are the optical representation of the supernatant plasma layer. The blood/plasma boundary is clearly seen. The random intensity fluctuations manifest a phase of the red blood. 
We will show that in this phase the rouleau formation process occurs. The rows of the intensity profiles characterize the other phase of the red blood. These rows reflect large wavelength density variations of the blood. Initially the blood was homogeneous thus the rows manifest a demixing process. The boundary moving from the bottom to the top of the container separates the two phases of the red blood. From Fig. 2 we also can see that the optical properties of the blood are dependent on the hematocrit. The repeatable features of the optical representation of the blood sedimentation process are clearly seen.

(a)
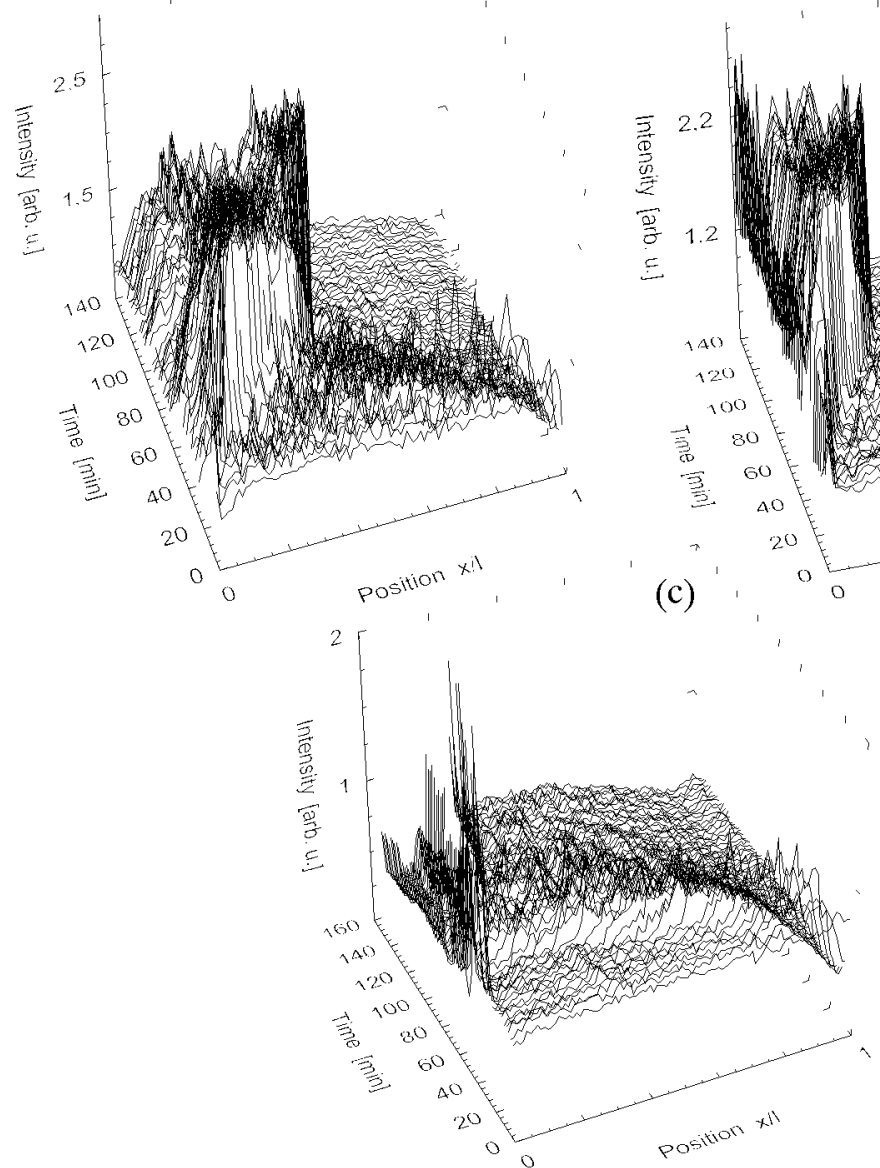

Fig. 2. Intensity profiles of light transmitted by the blood samples as a function of time at hematocrit $33 \%$ (a), $40 \%$ (b), and $57 \%$ (c). (b)

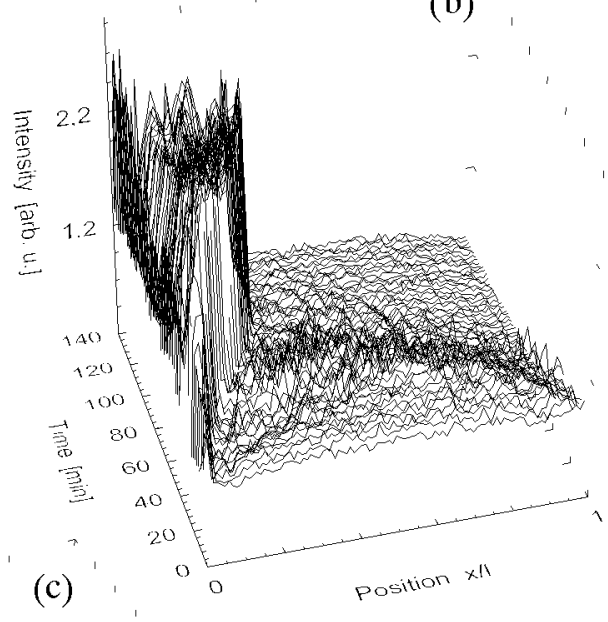


Figure 3 shows the temporal changes of the transmitted light intensity registered at a position $x / l=0.2$. The relatively small values of the intensity are the result of the scattering by the red blood. The sudden increase in this intensity indicates that at the moment the light starts to pass through plasma. The high transparency of plasma results in high values of the intensity. The instantaneous position of the blood/plasma boundary can be found with a relatively high accuracy. This figure shows also that the optical properties of the plasma change with time.
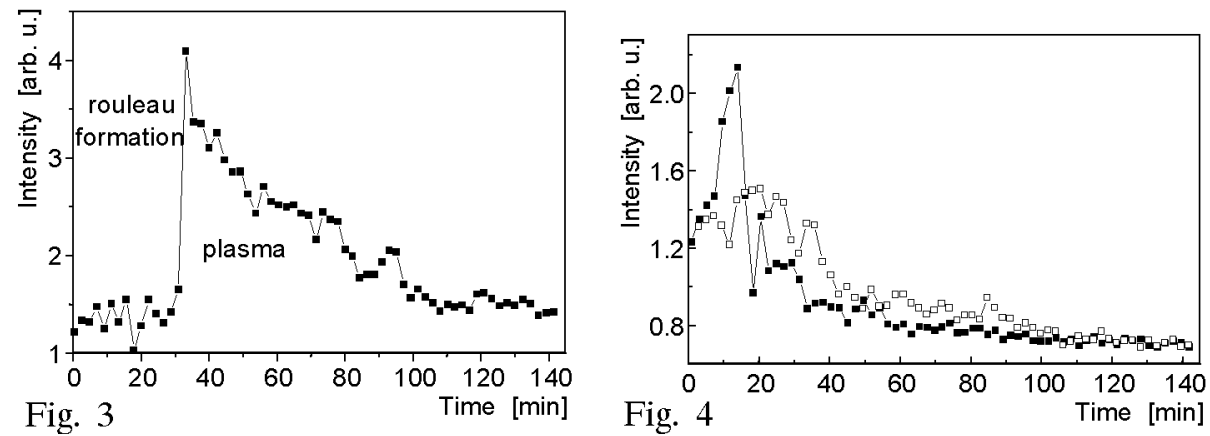

Fig. 3. The intensity of light transmitted by the blood sample as a function of time at a position of $x / l=0.2$. The hematocrit of the blood is $33 \%$.

Fig. 4. The intensity of light transmitted by the blood sample as a function of time at a position of $x / l=0.7(\mathbf{\square})$ and $x / l=0.55(\square)$. The hematocrit of the blood is $33 \%$.

To recognize the properties of the red blood the intensity profiles are analyzed. Figure 4 shows the changes of the transmitted light intensity as a function of time. At a position of $x / l=0.7$ this intensity first increases, next reaches a sharp maximum and finally decreases. The increasing intensity reflects the presence of the rouleau formation phase of the red blood. This temporal increase in the transmitted intensity is the well-known feature of the rouleau formation process. The obtained data are insufficient to determine the kinetics of the rouleau formation. The sudden decrease precedes the slow decreasing of the intensity. This slow decrease corresponds to the demixed phase of the blood. At a position of $x / l=0.55$ the intensity maximum becomes broad and a sudden decrease is not observed. Thus the temporal dependence of the intensity permits us to find the boundary between the two phases of the red blood in the early stages of the sedimentation process only. Figure 5 shows the intensity of the transmitted light as a function of the position received at 26 min from the beginning of the sedimentation process. This intensity profile can be fitted with two straight lines. This fit represents a first approximation of the optical representation of the process. The first line corresponds to the rouleau formation phase. The process of the rouleau formation occurring in this phase produces large intensity fluctuations. The intensity has a 


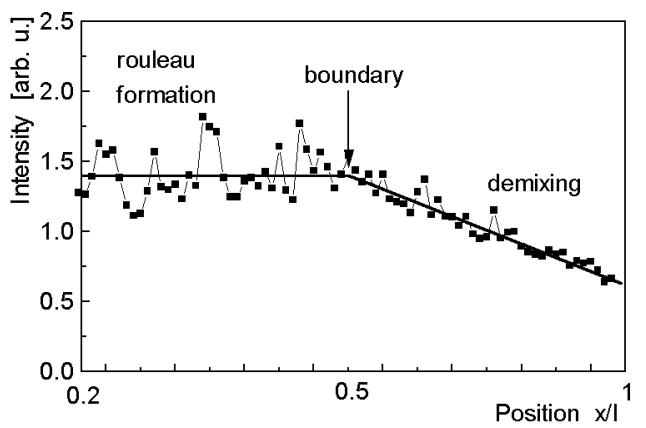

Fig. 5. Intensity profile of light transmitted by the blood sample. The hematocrit of the blood is $33 \%$. The straight lines represent the fit described in the text.
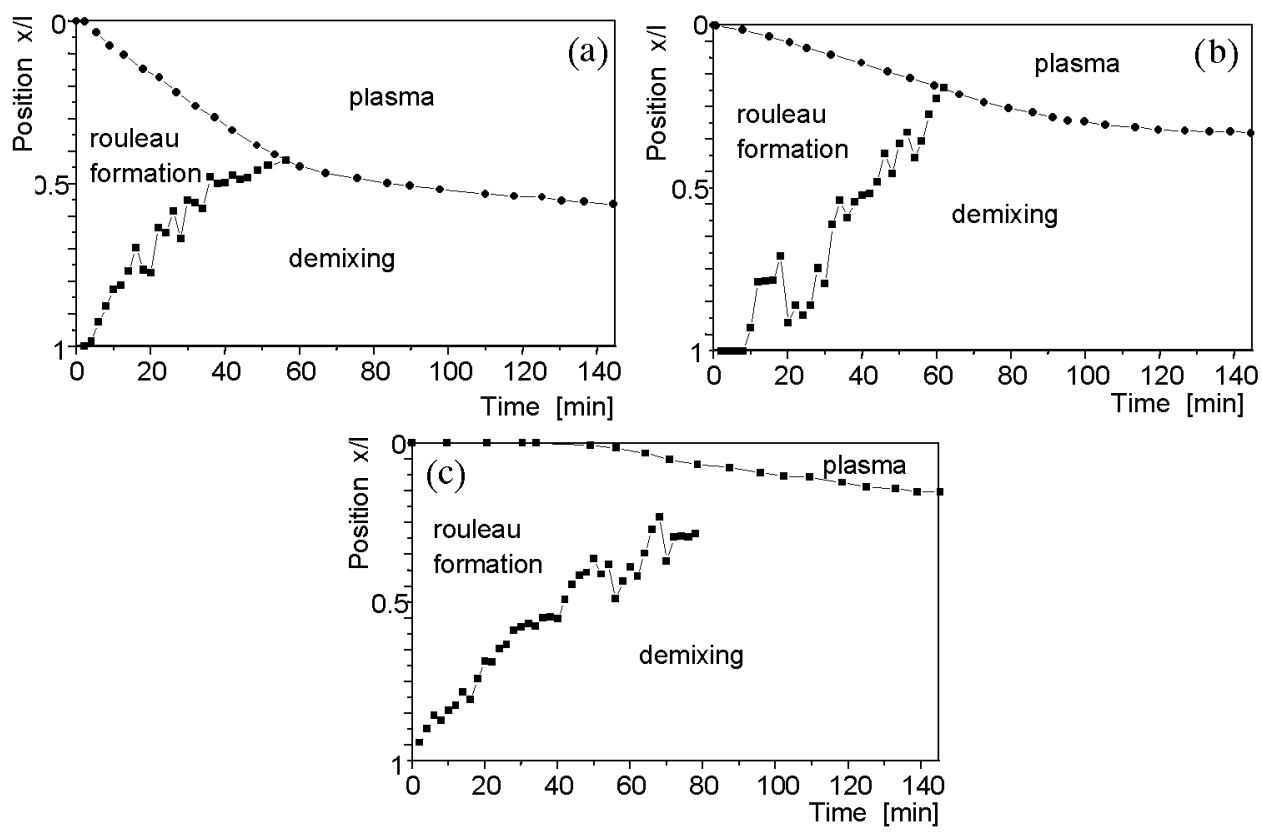

Fig. 6. The kinetics of the boundaries found in the human blood sediment at hematocrit $33 \%$ (a), $40 \%$ (b), and $57 \%$ (c).

constant value along the region occupied by this phase. The second line corresponds to the demixed phase of the red blood. The decrease in the intensity along the region occupied by the second phase reflects an increase in the concentration of the scattering units of this phase. The two lines join at a position. This position defines the instantaneous position of the boundary between the two phases. This simple scheme is applicable to the obtained intensity profiles. By the peak to peak analysis we have found that the distance between the rows of the intensity profiles ranges from $0.7 \mathrm{~mm}$ to $1.6 \mathrm{~mm}$. No relation was found between this distance and 
the hematocrit of the blood.

Figure 6 shows the kinetics of the boundaries found in the sediment of the blood. The boundaries separate the plasma and the two phases of the red blood. For the blood with the hematocrit $57 \%$ we were able to determine a part of the kinetics only. In the first period of the sedimentation process the plasma, the rouleau formation phase, and the demixed phase are present in the sediment. With time the rouleau formation phase reduces and disappears. In the second period of the process the sediment consists of the plasma and the demixed phase. The time at which the rouleau formation phase disappears increases as the blood hemotocrit increases.

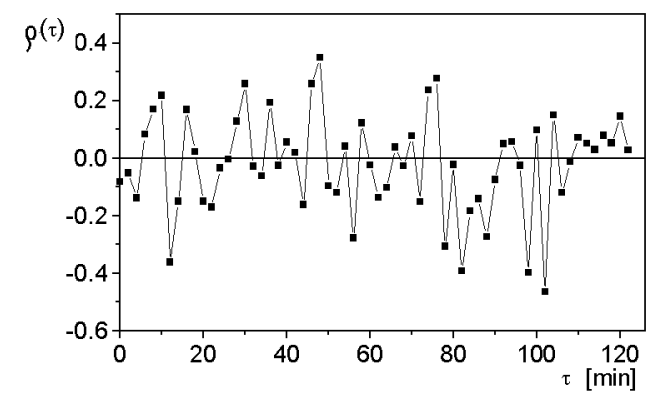

Fig. 7. Correlation coefficients of the intensity profile deviations at hematocrit $33 \%$.

We have analyzed dependences between the intensity profiles corresponding to the rouleau formation phase and the profiles of the demixed phase to verify that the sample preparation does not affect our results. Averaged intensities of the profiles were calculated and subtracted from the signals. In this way we have obtained a set of series of the intensity deviation. The correlation coefficients $\rho(\tau)$ calculated from the first and successive series of the intensity deviation are a measure of the similarity of the fluctuations. Obtained in this way temporal dependence of the correlation coefficient is shown in Fig. 7. From this figure one can see that the intensity deviations are uncorrelated. It confirms that the blood demixing is the result of the sedimentation process.

\section{Discussion}

In this paper we have extended the conventional approach in the study of blood sedimentation by the analysis of the optical properties of the blood sediment. This study permits us to grasp some physical mechanisms of the blood sedimentation process. Indeed, we have arrived at the set of experimental results that suits an approximate and qualitative analysis of the blood sedimentation mechanisms. Our experimental data are an optical representation of the blood states however we 
are unable to adopt some laws to support the analysis. Despite the limitations the received data are admission to the discussion of the blood sedimentation process.

The optical properties of the samples permit us to distinguish three phases of the sediment. The supernatant plasma layer creates at the top of the container. The plasma is not homogeneous and in the phase a time dependent process occurs. The plasma and the red blood are separated by the well-known blood/plasma boundary. In the sediment of the red blood we have found the phase of rouleau formation and the phase of demixed blood. The boundary moving from the bottom to the top separates the two phases.

The position of the blood/plasma boundary can be obtained with a relatively high accuracy from the optical data. The temporal dependence of the blood/plasma boundary position gives the well-known erythrocyte sedimentation rate curve. Huang et al. described this curve with two straight lines and found that the second straight line corresponds to the phase of already formed aggregates [11]. We have found the another one boundary moving in the red blood sediment. The approximate kinetics of the boundary was determined. The kinetics shows that since the time when the two boundaries met, the demixed phase of the red blood exists in the sediment only. On the other hand, we have found that the second straight line of the erythrocyte sedimentation curve corresponds to the phase of the demixed blood. There is no contradiction between the finding of Huang et al. [11] and our result. In this paper the phase of already formed aggregates is called the phase of the demixed blood, because the optical data exhibit the demixing process.

Free erythrocytes, achieved by the mixing procedure, coalesce in the container to form rouleaux. The time necessary to an initial rouleau formation is of the order of $1 \mathrm{~min}$. This short-term process can be modeled by means of Smoluchowski's theory $[6,15]$. However, with time the formed rouleaux can dissociate and associate. An equilibrium state of the blood can occur as a result of the reversible reactions. Samsel and Perelson found the equilibrium solution of a reversible kinetic equation that describes the rouleau formation [3]. We have found that in the course of the sedimentation process the blood of the rouleau formation phase does not reach equilibrium. At the bottom part of the container the rouleau formation process is suddenly stopped and the demixed start to develop. With time the region occupied by this phase of the red blood reduces and disappears.

Finally let us consider the hypothesis about the phase separation of the blood during sedimentation. When an idealized colloidal system is quenched from a stable state to an unstable long ranged correlations start to develop. To reach the unstable state the system crosses the spinodal line. The spinodal decomposition kinetics can be described by the Cahn-Hillard theory. Demixing of the initially homogeneous system is a result of this decomposition $[8,16]$. The blood is a bio-colloidal system thus we hypothesize the following scenario for the sedimentation process. Initially homogeneous blood consisting of free erythrocytes reaches a quasistable state. Friction forces reduce the velocity of erythrocytes and the attractive interaction 
between erythrocytes causes the rouleau formation. At the bottom of the container the system is quenched to the unstable region of their phase diagram. As the result of the quench the long ranged correlations start to develop and the demixing of the blood occurs. The chaotic network formed by the rouleaux reduces the growth of the blood density fluctuations. In the next part of the blood this scenario is repeated. The phase separation of the whole red blood is finished when the highest part of the blood is quenched. The sedimentation of the demixed blood occurs via destruction of the network. This model of the blood sedimentation is maybe too simple to be accurate, however highlight the experimental data.

\section{References}

[1] B.A. Schottelius, D.D. Schottelius, Textbook of Physiology, The C.V. Mosby Company, Saint Louis 1978.

[2] R.W. Samsel, A.S. Perelson, Biophys. J. 37, 493 (1982).

[3] R.W. Samsel, A.S. Perelson, Biophys. J. 45, 805 (1984).

[4] A.V. Priezzhev, O.M. Ryaboshapka, N.N. Firsov, I.V. Sirko, J. Biomed. Opt. 4, 76 (1999).

[5] A. Gaspar-Rosas, G.B. Thurston, Biorheolog. 25, 471 (1988).

[6] S.M. Bertoluzzo, A. Bollini, M. Rasia, A. Raynal, Blood Cells, Molecules, and Diseases 25, 339 (1999).

[7] V.L. Voeikov, Uspekhi Fiziol. Nauk. 29, 55 (1998) (in Russian).

[8] J.K.G. Dhont, An Introduction to Dynamics of Colloids, Elsevier, Amsterdam 1996.

[9] E. Biernacki, Z. F. Physiolog. Chem. 19, 179 (1894).

[10] V. Voeikov, S. Kondakov, E. Buravleva, I. Kaganovsky, M. Reznikov, SPIE Proc. 3923, 178 (2000).

[11] W.T. Hung, A.F. Collings, J. Low, Proc. Xth Int. Congr. Rheolog. 1, 425 (1988).

[12] V.V. Tuchin, X. Xu, R.K. Wang, J.B. Elder, Proc. SPIE 4263, 19 (2001).

[13] C.R. Huang, J.A. Whelan, H.H. Wang, A.L. Copley, Biorheolog. 8, 157 (1971).

[14] A.J. Reuben, A.G. Shannon, J. Math. Appl. Med. Biol. 7, 145 (1990).

[15] M.V. Smoluchowski, Z. Phys. Chem. 92, 129 (1917).

[16] A.J. Wagner, Int. J. Mod. Phys. C 9, 1373 (1998). 